Discrete Comput Geom 26:173-181 (2001)

DOI: $10.1007 / \mathrm{s} 00454-001-0011-5$

\title{
A Smaller Sleeping Bag for a Baby Snake
}

\author{
J. Håstad, ${ }^{1}$ S. Linusson, ${ }^{2}$ and J. Wästlund ${ }^{2}$ \\ ${ }^{1}$ NADA, Royal Institute of Technology, \\ S-100 44 Stockholm, Sweden \\ johanh@nada.kth.se \\ ${ }^{2}$ Department of Mathematics, Linköping University, \\ S-581 83 Linköping, Sweden \\ linusson@mai.liu.se \\ johan.waestlund@telia.com
}

\begin{abstract}
By a sleeping bag for a baby snake in $d$ dimensions we mean a subset of $\mathbb{R}^{d}$ which can cover, by rotation and translation, every curve of unit length. We construct sleeping bags which are smaller than any previously known in dimensions 3 and higher. In particular, we construct a three-dimensional sleeping bag of volume approximately 0.075803 . For large $d$ we construct $d$-dimensional sleeping bags with volume less than $(c \sqrt{\log d})^{d} / d^{3 d / 2}$ for some constant $c$.

To obtain the last result, we show that every curve of unit length in $\mathbb{R}^{d}$ lies between two parallel hyperplanes at distance at most $c_{1} d^{-3 / 2} \sqrt{\log d}$, for some constant $c_{1}$.
\end{abstract}

\section{Introduction}

The "worm problem" of Leo Moser asks for a plane set of minimal area which can cover (by rotation and translation) every arc of unit length. The problem has been popularized by several authors, see [St], [NPL] and the references therein. It has been referred to as "mother worm's blanket", while other mathematicians have discussed the form of a hammer head to smash the entire worm in a single stroke.

The smallest known "universal blanket" was discovered by Norwood et al. [NPL]. This region, shown in Fig. 1, consists of a $60^{\circ}$-sector of a circle of radius $\frac{1}{2}$, with a $30^{\circ}-60^{\circ}-90^{\circ}$ triangle with sides $\frac{1}{2}, \sqrt{3} / 3$ and $\sqrt{3} / 6$ attached on each side. The area is $\sqrt{3} / 12+\pi / 24$, or approximately 0.27524 . No positive lower bound is known for the area of a universal blanket, although in $[\mathrm{M}]$ it is shown that no plane set of measure zero will cover every smooth curve. If we add the requirement that the blanket should be convex, then it is shown in [SW] that the area must be at least 0.21946 . 


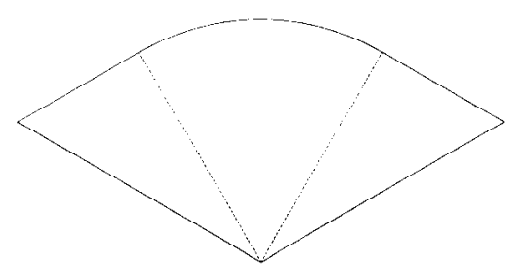

Fig. 1. The smallest known universal blanket.

In [St], Stewart asks for a "sleeping bag", that is, a three-dimensional region which will cover every arc of unit length. This challenge was answered by Lindström [L], who constructed a valid sleeping bag of approximate volume 0.15953 . In this note we construct a sleeping bag in three dimensions with less than half the volume of Lindström's. We also construct higher-dimensional sleeping bags which, as far as we know, are smaller than any previously known, also asymptotically. The higher-dimensional problem has also been studied by Schaer and Wetzel [SW], who proved that the cube with hyperdiagonal 1 is a valid sleeping bag in every dimension.

We begin by constructing a sleeping bag with the shape of a rectangular box.

Theorem 1. The rectangular $1 \times \frac{1}{2} \times \frac{1}{3} \times \cdots \times 1 / d$ box is a valid sleeping bag in $d$ dimensions.

Proof. Suppose we are given an arc $\gamma$ of unit length in $d$ dimensions. We can assume that $\gamma:[0,1] \rightarrow \mathbb{R}^{d}$ is a parametrization by arc length, that is, the $\operatorname{arc} \gamma[a, b]$ has length $|a-b|$. Let $H$ be a hyperplane intersecting $\gamma$ in the points $\gamma(1 / 2 d), \gamma(3 / 2 d), \ldots$, $\gamma((2 d-1) / 2 d)$. Since no point on $\gamma$ can be at a distance more than $1 / 2 d$ from $H, \gamma$ lies completely between the two hyperplanes parallel to $H$ at a distance $1 / 2 d$ from $H$. These two hyperplanes, which we call $H_{1}$ and $H_{2}$, are at a distance $1 / d$ from each other. By induction on $d$, we can assume that the projection of $\gamma$ on $H$ can be covered by a $1 \times \frac{1}{2} \times \frac{1}{3} \times \cdots \times 1 /(d-1)$ box. The part of the region between $H_{1}$ and $H_{2}$ which is projected into this box by orthogonal projection on $H$ is a $1 \times \frac{1}{2} \times \frac{1}{3} \times \cdots \times 1 / d$ box containing $\gamma$.

This box has volume $1 / d$ !, which is smaller than the volume $1 / d^{d / 2}$ of the cube with diagonal 1 (see Table 1). We make two different refinements of this construction. The first one gives slightly smaller sleeping bags in low dimensions, by removing some parts of the box in Theorem 1 . The second one gives considerably smaller rectangular boxes in higher dimensions.

We use the following notation. Let $\gamma$ be a curve in $\mathbb{R}^{d}$ and let $\pi$ be a projection of $\mathbb{R}^{d}$ to $\mathbb{R}$. Then we let $w_{\pi}(\gamma)$ be the length of the interval $\pi(\gamma)$. For a hyperplane $H$ we let $w_{H}(\gamma)=w_{\pi}(\gamma)$ where $\pi$ is the orthogonal projection projecting $H$ to a single point. We let the width $w(\gamma)$ of $\gamma$ be $\min _{\pi} w_{\pi}(\gamma)$. Finally, we let $t(d)$ be the supremum of $w(\gamma)$ over all curves $\gamma$ of length 1 in $\mathbb{R}^{d}$. Theorem 1 is a combination of the two facts that $t(d) \leq d^{-1}$ and that there is a rectangular sleeping bag of volume $\prod_{i=1}^{d} t(i)$. In 
Section 3 we prove that

$$
d^{-3 / 2} \leq t(d) \leq \frac{\frac{9}{2} \sqrt{\log d}+1}{(d-3)^{3 / 2}} .
$$

\section{A Construction for Small Sleeping Bags in Low Dimensions}

We can reduce the volume of the sleeping bag in Theorem 1 by a constant factor by taking the blanket of Norwood et al. as the starting point of the induction. The following theorem makes a more substantial improvement on this construction by rounding off the corners of the box. Recall that a set $S$ is star convex if there is a central point $p \in S$ such that for every point $x \in S$, the line segment from $p$ to $x$ lies entirely in $S$.

Theorem 2. Let $R$ be a star convex sleeping bag ind -1 dimensions. Let $p$ be a central point of $R$. Let $R^{\prime}$ be the subset of $\mathbb{R}^{d}$ contained between the hyperplanes $x_{d}=0$ and $x_{d}=1 / d$, such that the intersection of $R^{\prime}$ with the hyperplane $x_{d}=h$ is a copy of $R$ scaled down by a factor

$$
1-\frac{1}{d}+\sqrt{\frac{1}{d^{2}}-h^{2}}
$$

around $p$. Then $R^{\prime}$ is a valid sleeping bag in d dimensions.

Proof. Let $\gamma$ be a curve in $\mathbb{R}^{d}$ of unit length parameterized by arc length. As in the proof of Theorem 1, let $H$ be a hyperplane passing through the points $\gamma(1 / 2 d), \gamma(3 / 2 d), \ldots$, $\gamma((2 d-1) / 2 d)$. Let $H_{1}$ and $H_{2}$ be the hyperplanes parallel to $H$ touching $\gamma$, such that $\gamma$ lies between them. Clearly, $w_{H}(\gamma)$ is the distance between $H_{1}$ and $H_{2}$, and for notational convenience we denote this number by $w$. We let $\ell$ denote the length of the projection of $\gamma$ on $H$ and we start by deriving an inequality relating $w$ to $\ell$.

Let $w_{1}$ and $w_{2}$ be the distances from $H$ to $H_{1}$ and $H_{2}$, respectively. We divide $\gamma$ into $2 d \operatorname{arcs} \gamma_{1}, \ldots, \gamma_{2 d}$ of equal length. Note that each of them has an endpoint in $H$. If the arc $\gamma_{k}=\gamma[(k-1) / 2 d, k / 2 d]$ contains a point at distance $w_{1}$ from $H$, then the length $\ell\left(\gamma_{k}\right)$ of the projection of $\gamma_{k}$ on $H$ is at most

$$
\sqrt{\frac{1}{4 d^{2}}-w_{1}^{2}}
$$

There is also an arc $\gamma_{l}$ containing a point at distance $w_{2}$ from $H$ on the other side of $H$. The sum $w\left(\gamma_{k}\right)+w\left(\gamma_{l}\right)$ of the lengths of the projections of $\gamma_{k}$ and $\gamma_{l}$ on $H$ is at most

$$
\sqrt{\frac{1}{4 d^{2}}-w_{1}^{2}}+\sqrt{\frac{1}{4 d^{2}}-w_{2}^{2}}
$$

Under the condition that $w_{1}+w_{2}=w$, this is maximized by taking $w_{1}=w_{2}$, so that 1 becomes

$$
\sqrt{\frac{1}{d^{2}}-w^{2}}
$$


Under the assumption that $k \neq l$, it follows that

$$
\ell \leq 1-\frac{1}{d}+\sqrt{\frac{1}{d^{2}}-w^{2}} .
$$

We will show that $w$ cannot be greater than this even if $k=l$. Even without taking into account that $\gamma_{k}$ must return to $H$ after visiting $H_{1}$ and $H_{2}$, we get

$$
\begin{aligned}
\ell & \leq 1-\frac{1}{2 d}+\sqrt{\frac{1}{4 d^{2}}-w^{2}}=1-\frac{1}{d}+\sqrt{\frac{1}{4 d^{2}}-w^{2}}+\frac{1}{2 d} \\
& =1-\frac{1}{d}+\sqrt{\frac{1}{2 d^{2}}-w^{2}+\frac{1}{d} \sqrt{\frac{1}{4 d^{2}}-w^{2}}} \leq 1-\frac{1}{d}+\sqrt{\frac{1}{2 d^{2}}-w^{2}+\frac{1}{d} \sqrt{\frac{1}{4 d^{2}}}} \\
& =1-\frac{1}{d}+\sqrt{\frac{1}{d^{2}}-w^{2} .}
\end{aligned}
$$

Hence the projection of $\gamma$ on $H$ can be covered by a copy $R_{w}$ of $R$ scaled down by a factor

$$
1-\frac{1}{d}+\sqrt{\frac{1}{d^{2}}-w^{2}} .
$$

This shows that $\gamma$ can always be covered by a prism over $R_{w}$ of width $w$. Since $R$ is star convex, this prism, and hence $\gamma$, can be covered by $R^{\prime}$.

We now compute the volume of the sleeping bag obtained in Theorem 2 . The volume of $R_{w}$ is

$$
\operatorname{vol}(R) \cdot\left(1-\frac{1}{d}+\sqrt{\frac{1}{d^{2}}-w^{2}}\right)^{d-1}
$$

Hence

$$
\begin{aligned}
\operatorname{vol}\left(R^{\prime}\right) & =\operatorname{vol}(R) \cdot \int_{0}^{1 / d}\left(1-\frac{1}{d}+\sqrt{\frac{1}{d^{2}}-w^{2}}\right)^{d-1} d w \\
& =\operatorname{vol}(R) \cdot \frac{1}{d} \cdot \int_{0}^{1}\left(1-\frac{1}{d}\left(1-\sqrt{1-x^{2}}\right)\right)^{d-1} d x
\end{aligned}
$$

by the substitution $w=x / d$.

The integral in 2 can of course be evaluated for any $d$. If we let $R$ be the twodimensional blanket of Norwood et al., then Theorem 2 gives us a three-dimensional sleeping bag with volume

$$
\left(\frac{14}{81}+\frac{\pi}{27}\right)\left(\frac{\sqrt{3}}{12}+\frac{\pi}{24}\right)
$$

or about 0.079597 . 
We now estimate the quotient $\operatorname{vol}\left(R^{\prime}\right) / \operatorname{vol}(R)$ for large $d$. Using the inequality $1-y \leq$ $e^{-y}$, with $y=\left(1-\sqrt{1-x^{2}}\right) / d$, we obtain

$$
\left(1-\frac{1}{d}\left(1-\sqrt{1-x^{2}}\right)\right)^{d-1} \leq \frac{d}{d-1} e^{\sqrt{1-x^{2}}-1} .
$$

Hence

$$
\frac{\operatorname{vol}\left(R^{\prime}\right)}{\operatorname{vol}(R)} \leq \frac{1}{d-1} \int_{0}^{1} e^{\sqrt{1-x^{2}}-1} d x .
$$

Since $R^{\prime}$ is also star convex, it follows by induction on $d$ that there exists a $d$ dimensional valid sleeping bag with volume (at most)

$$
\frac{c^{d-1}}{(d-1) !}
$$

where

$$
c=\int_{0}^{1} e^{\sqrt{1-x^{2}}-1} d x \approx 0.82550 .
$$

An Even Smaller Sleeping Bag in $\mathbb{R}^{3}$

In three dimensions we can find an even smaller sleeping bag. Recall that $t(3)$ is the maximal width of any curve of length 1 in $\mathbb{R}^{3}$. Let $R$ be the two-dimensional blanket described by Norwood et al. pictured in Fig. 1 .

Theorem 3. The prism over $R$ scaled down by a factor $\sqrt{1-t(3)^{2}}$ and with height $t(3)$ is a valid sleeping bag. It has volume $t(3)\left(1-t(3)^{2}\right)(\sqrt{3} / 12+\pi / 24)$. In particular since $t(3) \leq 0.30331$, there is a valid sleeping bag in $\mathbb{R}^{3}$ with volume less than 0.075803 .

Proof. Let $\gamma$ be an arc in $\mathbb{R}^{3}$. If $w_{\pi}(\gamma) \leq t$ (3) for every direction $\pi$, then $\gamma$ fits in a cube with side $t$ (3). Since a square can easily be placed in $R$ as long as the diagonal is at most $\frac{1}{2}$, the cube will fit in the described prism as long as $\frac{1}{2} \sqrt{1-t(3)^{2}} \geq t(3) \sqrt{2}$. This is true if $t(3) \leq \frac{1}{3}$, which we already know is the case.

Suppose therefore that there is a projection $\pi^{\prime}$ for which $w_{\pi^{\prime}}(\gamma)>t(3)$. By continuity there is a $\pi$ for which $w_{\pi}(\gamma)=t(3)$. Now the projection of $\gamma$ to the hyperplane orthogonal to $\pi$ is a two-dimensional arc of length at most $\sqrt{1-t(3)}$, which will fit in a copy of $R$ scaled down by the same factor. It is now clear that all of $\gamma$ can be fitted into the given prism.

The last statement of the theorem follows from the lemma below.

\section{Lemma 4.}

$$
t(3) \leq \frac{1}{\sqrt{1+\pi^{2}}}
$$


Table 1. Comparison of the volumes of some sleeping bags.

\begin{tabular}{lccccc}
\hline & \multicolumn{5}{c}{ Dimension } \\
\cline { 2 - 6 } Method & \multicolumn{1}{c}{3} & 4 & 5 & 6 & 7 \\
\hline The cube with diagonal 1 & 0.19245 & 0.0625 & 0.017889 & $4.63 \cdot 10^{-3}$ & $1.10 \cdot 10^{-3}$ \\
Lindström's sleeping bag & 0.15953 & & & & \\
The box of Theorem 1 & 0.16667 & 0.041667 & $8.33 \cdot 10^{-3}$ & $1.39 \cdot 10^{-3}$ & $1.98 \cdot 10^{-4}$ \\
The rounded prism of Theorem 2 & 0.079597 & 0.017037 & $2.90 \cdot 10^{-3}$ & $4.08 \cdot 10^{-4}$ & $4.91 \cdot 10^{-5}$ \\
The prism of Theorem 3 & 0.075803 & & & & \\
\hline
\end{tabular}

Proof. Let $\gamma$ be a curve of unit length in $\mathbb{R}^{3}$. Let $\sigma$ be the orthogonal projection to the line through the endpoints of $\gamma$, and let $H$ be a plane orthogonal to this line. Let $w=w_{\sigma}(\gamma)$. The length of the projection of $\gamma$ to $H$ is at most $\sqrt{1-w^{2}}$. Note that the projection of $\gamma$ to $H$ is a closed curve.

It is a well known fact from convex geometry (see for example p. 6 of [Sa]) that the average width, taken over all directions, of a closed curve of unit length in two dimensions is at most $1 / \pi$ (with equality if the curve equals the boundary of its convex hull).

Hence in some direction, the width of $\gamma$ is at most $\sqrt{1-w^{2}} / \pi$. We therefore have

$$
w(\gamma) \leq \min \left(w, \frac{\sqrt{1-w^{2}}}{\pi}\right) .
$$

To get an upper bound for the right-hand side of 3 , we put $w=\sqrt{1-w^{2}} / \pi$, and solve for $w$, to get $w=1 / \sqrt{1+\pi^{2}}$.

Table 1 compares the different constructions.

\section{Bounds for $t(d)$ in Higher Dimensions}

In this section we estimate $t(d)$ for large $d$. Apart from the immediate application to the sleeping bag problem we believe that the estimation of $t(d)$ is an interesting problem in itself. An exact calculation of $t$ (2) (approximately 0.438925) can be found in [Sc]. A description of the curve giving the lower bound on $t(2)$ can be found in [SW].

Theorem 5. $t(d) \geq d^{-3 / 2}$

Proof. Take the curve $\gamma$ that consists of $d$ segments of length $1 / d$, where the $i$ th segment is in the direction of the $i$ th coordinate axis. Now take any linear function $\pi: \mathbb{R}^{d} \rightarrow \mathbb{R}$, $\pi\left(x_{1}, x_{2}, \ldots, x_{d}\right)=\sum_{i=1}^{d} a_{i} x_{i}$, where $\sum_{i=1}^{d} a_{i}^{2}=1$. There has to be some $a_{i} \geq d^{-1 / 2}$. This means that the $i$ th segment of $\gamma$ has a projection of size at least $d^{-3 / 2}$.

The rest of the section is devoted to the following upper bound on $t(d)$. 


\section{Theorem 6.}

$$
t(d) \leq \frac{\frac{9}{2} \sqrt{\log d}+1}{(d-3)^{3 / 2}} .
$$

We need a few preliminary results.

Lemma 7. The surface area of the spherical cap defined by $x_{1}>c,\|x\|=1$ in $\mathbb{R}^{d}$, equals

$$
\frac{2 \pi^{(d-1) / 2}}{\Gamma((d-1) / 2)} \int_{c}^{1}\left(1-x^{2}\right)^{(d-3) / 2} d x .
$$

Proof. We use the fact that the volume of the $(d-1)$-dimensional unit ball equals

$$
\frac{2 \pi^{(d-1) / 2}}{(d-1) \Gamma((d-1) / 2)} .
$$

The surface area in question equals $d$ times the volume of the region in $\mathbb{R}^{d}$ defined by $\|x\| \leq 1, x_{1} /\|x\| \geq c$. This is

$$
\begin{aligned}
& \frac{2 d \pi^{(d-1) / 2}}{(d-1) \Gamma((d-1) / 2)}\left(\int_{0}^{c}\left(\frac{\sqrt{1-c^{2}}}{c} x\right)^{d-1} d x+\int_{c}^{1}\left(\sqrt{1-x^{2}}\right)^{d-1} d x\right) \\
& =\frac{2 \pi^{(d-1) / 2}}{(d-1) \Gamma((d-1) / 2)}\left(c\left(1-c^{2}\right)^{(d-1) / 2}+d \int_{c}^{1}\left(1-x^{2}\right)^{(d-1) / 2} d x\right) .
\end{aligned}
$$

Differentiating the second factor of 4 with respect to $c$, we obtain

$$
\left(1-c^{2}\right)^{(d-3) / 2}\left(1-d c^{2}\right)-d\left(1-c^{2}\right)^{(d-1) / 2},
$$

which simplifies to $(1-d)\left(1-c^{2}\right)^{(d-3) / 2}$. Hence 4 equals

$$
\frac{2 \pi^{(d-1) / 2}}{\Gamma((d-1) / 2)} \int_{c}^{1}\left(1-x^{2}\right)^{(d-3) / 2} d x+\text { constant } .
$$

Since both 4 and 5 tend to 0 as $c \rightarrow 1$, the constant is zero.

Lemma 8. Suppose $x \in \mathbb{R}^{d}, c \in[0,1]$ and let $\pi$ be a random projection from $\mathbb{R}^{d}$ to $\mathbb{R}$. Then

$$
\operatorname{Pr}(|\pi x| \geq c\|x\|) \leq\left(1-c^{2}\right)^{(d-3) / 2}
$$

Proof. By symmetry we can clearly assume that $x=e_{1}$, the unit vector in the first dimension. The surface area of the part of $S^{d}$ with $x_{1}>c$ is equal to

$$
\frac{2 \pi^{(d-1) / 2}}{\Gamma((d-1) / 2)} \int_{c}^{1}\left(1-x^{2}\right)^{(d-3) / 2} d x .
$$


Hence

$$
\begin{aligned}
\operatorname{Pr}(|\pi x| \geq c\|x\|) & =\frac{\int_{c}^{1}\left(1-x^{2}\right)^{(d-3) / 2} d x}{\int_{0}^{1}\left(1-x^{2}\right)^{(d-3) / 2} d x} \leq \frac{\int_{c}^{1}\left(1-x^{2}\right)^{(d-3) / 2} d x}{\int_{0}^{1-c}\left(1-x^{2}\right)^{(d-3) / 2} d x} \\
& =\frac{\int_{0}^{1-c}\left(1-(x+c)^{2}\right)^{(d-3) / 2} d x}{\int_{0}^{1-c}\left(1-x^{2}\right)^{(d-3) / 2} d x} \leq\left(1-c^{2}\right)^{(d-3) / 2},
\end{aligned}
$$

the maximal value of the quotient of the integrands.

As in the proof of Theorem 1, let $\gamma$ be a curve parameterized by arc length. We choose two integers $d_{1}$ and $d_{2}$, to be specified later, such that $d_{1}+d_{2}=d+1$. Take an orthogonal linear projection $\tau$ that projects $\mathbb{R}^{d}$ to $\mathbb{R}^{d_{2}}$, such that all $\gamma\left((2 i+1) / 2 d_{1}\right)$, $i=0, \ldots, d_{1}-1$, projects to the same point, which we can assume to be the origin. We compose this projection with a random projection $\sigma$ from $\mathbb{R}^{d_{2}}$ to $\mathbb{R}$. We analyze the combined projection $\pi=\sigma \circ \tau$.

Lemma 9. Let $p$ be any point on $\gamma$, and let $h>0$. Then

$$
\operatorname{Pr}(|\pi(p)|>h) \leq\left(1-4 d_{1}^{2} h^{2}\right)^{\left(d_{2}-3\right) / 2} .
$$

Proof. Note that the distance from $p$ to the nearest point $\gamma\left((2 i+1) / 2 d_{1}\right)$ is at most $1 / 2 d_{1}$. We now apply Lemma 8 (in $d_{2}$ dimensions).

Proof of Theorem 6. We now divide the curve $\gamma$ into $N$ parts. The probability that at least one of the midpoints of these parts is mapped by $\pi$ to a point at distance more than $h$ from the origin is at most

$$
N \cdot\left(1-4 d_{1}^{2} h^{2}\right)^{\left(d_{2}-3\right) / 2} .
$$

If $d_{1}, d_{2}, N$ and $h$ are chosen so that

$$
1-N\left(1-4 d_{1}^{2} h^{2}\right)^{\left(d_{2}-3\right) / 2}>0,
$$

then with positive probability none of the points $\pi(\gamma((2 i+1) / 2 N))$ is at a distance more than $h$ from the origin, and hence no point on $\pi(\gamma)$ is at a distance more than $h+1 / 2 N$ from the origin. It will follow that $t(d) \leq 2 h+1 / N$.

By continuity, having chosen $d_{1}, d_{2}$ and $N$, we can choose $h$ so that

$$
1-N\left(1-4 d_{1}^{2} h^{2}\right)^{\left(d_{2}-3\right) / 2}=0 .
$$

Putting $d_{2}=d-d_{1}+1$, and solving for $h$, we get

$$
h=\frac{\sqrt{1-N^{-2 /\left(d-d_{1}-2\right)}},}{2 d_{1}},
$$

and, consequently,

$$
t(d) \leq \frac{\sqrt{1-N^{-2 /\left(d-d_{1}-2\right)}}}{d_{1}}+\frac{1}{N} .
$$


Using the inequality $1-e^{-x} \leq x$, with $x=\log N \cdot 2 /\left(d-d_{1}-2\right)$, we obtain

$$
t(d) \leq \frac{\sqrt{\log N \cdot 2 /\left(d-d_{1}-2\right)}}{d_{1}}+\frac{1}{N}=\sqrt{\log N} \cdot \sqrt{\frac{2}{d_{1}^{2}\left(d-2-d_{1}\right)}}+\frac{1}{N} .
$$

We now optimize our choice of $d_{1}$ and $d_{2}$. To maximize $d_{1}^{2}\left(d-2-d_{1}\right)$, we choose $d_{1} \approx \frac{2}{3} d$. We have to take into account that $d_{1}$ has to be an integer, but in any case we can make sure that $d_{1}^{2}\left(d-2-d_{1}\right) \geq \frac{4}{27}(d-3)^{3}$.

We then have

$$
t(d) \leq \sqrt{\frac{27}{2(d-3)^{3}}} \sqrt{\log N}+\frac{1}{N} .
$$

We now choose $N$ to be $d^{3 / 2}$ rounded to the nearest integer. This is slightly smaller than the optimal value, but the exponent $\frac{3}{2}$ is the best one.

We obtain

$$
t(d) \leq \sqrt{\frac{27}{2(d-3)^{3}}} \sqrt{\log d^{3 / 2}}+\frac{1}{(d-1)^{3 / 2}} \leq \frac{\frac{9}{2} \sqrt{\log d}+1}{(d-3)^{3 / 2}} .
$$

This gives a better bound than that of Theorem 1 when $d \geq 128$. Theorem 5 immediately gives the following corollary:

Corollary 10. The rectangular $t(1) \times t(2) \times \cdots \times t(d)$ box has volume less than

$$
\frac{(c \sqrt{\log d})^{d}}{d^{3 d / 2}},
$$

for some constant $c$.

\section{Acknowledgment}

The authors thank Bernt Lindström for introducing us to the subject.

\section{References}

[L] B. Lindström, A sleeping bag for a baby snake, Math. Gaz. 81(492) (1997), 451-452.

[M] J. M. Marstrand, Packing smooth curves in $\mathbb{R}^{q}$, Mathematika 26 (1979), 1-12.

[NPL] R. Norwood, G. Poole, and M. Laidacker, The worm problem of Leo Moser, Discrete Comput. Geom. 7 (1992), 153-162.

[Sa] L. A. Santaló, Encyclopedia of Mathematics, Vol. I: Integral Geometry and Geometric Probability, Addison-Wesley, Reading, MA, 1976.

[Sc] J. Schaer, The boadest curve of length 1, Research Paper No. 52, Department of Mathematics, Statistics, and Computer Science, University of Calgary, 1968.

[SW] J. Schaer and J. E. Wetzel, Boxes for curves of constant length, Israel J. Math. 12 (1972), 257-265.

[St] I. Stewart, Game, Set and Math, Blackwell, London, 1989.

Received March 24, 1999, and in revised form July 26, 2000. Online publication April 6, 2001. 